\title{
Study on the Technology of Ultra-high Sensitive Wide-band Magnetic-feedback Inductive Magnetic Sensor
}

\author{
Yong Liu*, Wenbin Li, Jinrong Zhou, Rui Pan, Huan Zheng, Bing Xiang, and Rui Xu \\ LF Electromagnetic Communication Laboratory, Wuhan Maritime Communication Research Institute, 430205 Wuhan, China
}

\begin{abstract}
It analyses the composition and principle of high-sensitive wide-band magnetic-feedback inductive magnetic sensor to fulfil the demand of high-sensitive wide-band magnetic sensor in geological exploration. It studies main factors to the performance of wide-band magnetic sensor, such as turns of coils, core material features, and amplifier noise, specifies section-wise coil winding, the type and dimension of core material, and designs low-noise high-impedance LF chopping amplifier channel and composite amplifier with HF amplifier channel. The noise of magnetic sensor at $1 \mathrm{~Hz}$ is better than $10-4 \mathrm{nT} / \mathrm{Hz} 1 / 2$, at $100 \mathrm{~Hz}-1 \mathrm{kHz}$ band the noise floor is close to SQUID which can reach $10-6 \mathrm{nT} / \mathrm{Hz} 1 / 2$. The magnetic sensor works at wide frequency band $(0.0001 \mathrm{~Hz}-10 \mathrm{kHz})$ and ultra-low noise, which can meet the requirements of both AMT and CSAMT.
\end{abstract}

\section{Introduction}

High-sensitive wide-band inductive magnetic sensor is the most widely-applied magnetic receiver in electromagnetic prospecting, mainly used in the exploration of metal mineral, petroleum resources, and indepth geophysics[1-3].Electromagnetic prospecting is divided into audio frequency magnetelluric (AMT) and Controlled source audio frequency magnetelluric (CSAMT), with the former requiring high-sensitive magnetic sensor, and the latter wide frequency-band, meanwhile the sensor shall fulfil field requirements like small size, light weight, and low power consumption[2]. Based on actual field environment, the paper studies how to realize wide frequency-band and low-noise of inductive magnetic sensor from theoretical and field design perspective, which can fulfil the requirements of both AMT and CSAMT exploration.

\section{Composition and principle}

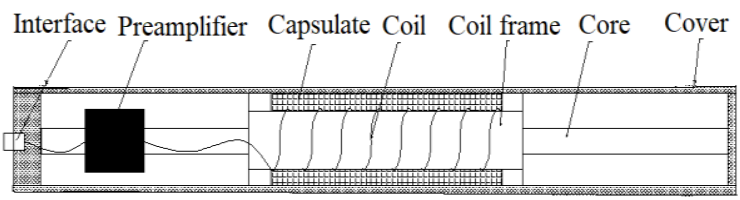

Fig. 1. Schematic of magnetic sensor composition.

Magnetic-feedback inductive magnetic sensor is mainly composed of core, coil (inductive coil and feedback coil included), pre-amplifier, coil frame, interface and insulating shell, the schematic of which is shown as Figure 1. The sensor is capsulated in the insulated cylinder with water-tightness and vibration-proof features, suitable for field environment and preventing water and mechanical shock force.

The basic principle of magnetic sensor is Faraday's law of electromagnetic induction, and inducted electromotive force $U_{i}$ will be generated in case that cylinder coil, with $N$ being the number of turns and $S$ being sectional area, is placed in the magnetic field $B(t)$ varied with time, which is shown as follows:

$$
U_{i}=-N S \frac{d B(t)}{d(t)}=-j \omega N S \mu_{r} B_{0}
$$

In the formula $N$ is the number of turns, $S$ sectional area, $\mu_{r}$ relative permeability of core, and $B_{0}$ being magnetic field component in the direction of core.

It is known from formula (1) that the increase of $N, S$, $\mu_{r}$ will directly enhance field induction capacity of coil [4], meanwhile reducing the local noise can enhance SNR of output signal, and raise magnetic induction capacity indirectly.

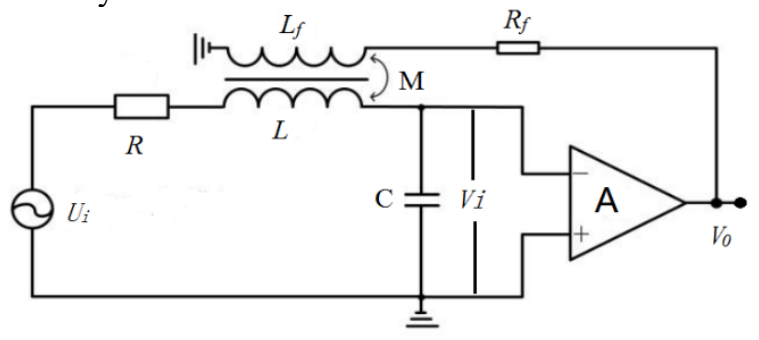

Fig. 2. Schematic of magnetic feedback inductive sensor.

Electrical performance depends on core, coil (inductive coil and feedback coil included), and low noise pre-amplifier. As shown in Figure 2, $R, L, C$ respectively are resistance, inductance, and distributive capacitance of inductive coil. Through the amplification of amplifier $A$, 
inducted output voltage of $L$ is entered into $L_{f}$ via $R_{f}$, in which a feedback magnetic field is formulated when there is current passing by, therefore minus feedback is established against the tested magnetic field, and minus compensation is carried out over the output signal at frequency band adjacent of resonance point, so that amplitude curve is smooth and even for output signal at these frequencies, in an effort to enhance bandwidth of magnetic sensor [5]. Generally, feedback is adopted to spread the frequencies upward to $10 \mathrm{kHz}$ above, and chopping amplifier to spread the frequencies downward to $0.0001 \mathrm{~Hz}$. Magnetic feedback can reduce the effect of temperature, humidity, and other factors on the sensor, enhancing consistency and stability. Usually feedback coil serves as nominal coil for calibrating magnetic sensor in the field.

Generally a layer of feedback coil is winded on inducted coil, with its inductance being $L_{f}$, resistance being $R_{f}$, and mutual inductance being $M$ via formula $M=Q \sqrt{L * L_{f}}$; close coupling between inducted coil and feedback coil is in place with its factor $Q=1$, hence $M=\sqrt{L * L_{f}}$. And transfer function $H(\omega)$ is shown as follows suppose gain of amplifying circuit being $A$, and output being $V_{0}$ :

$$
H(\omega)=\frac{V_{o}}{B_{0}}=\frac{j \omega \mu_{r} N S A}{1-\omega^{2} L C+j \omega\left(R C+\frac{A M}{R_{f}}\right)}
$$

It is found that LF stop frequency of amplitudefrequency characteristic is $f_{l}=\frac{R_{f}}{2 \pi A M}$, and HF stop frequency being $f_{h}=\frac{A M}{2 \pi R_{f} L C}$ based on transfer function. Therefore under the situation that feedback resistance is certain, i.e. feedback depth being constant, and that amplifying product $A$ of amplifying circuit is increased, the more feedback factor $\alpha$ is, LF stop frequency will be decreased, and HF stop frequency will be increased, which is conducive for spreading the width of frequency band.

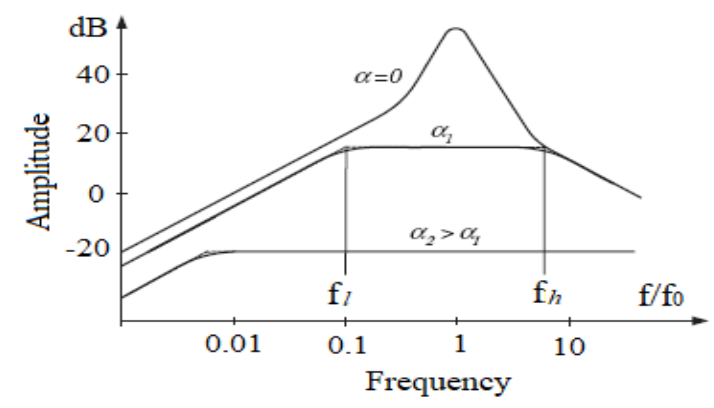

Fig. 3. Amplitude-frequency curve for magnetic sensor with different feedback factor $\alpha$.

Figure 3 shows amplitude-frequency curve for magnetic sensor with different feedback factor $\alpha$, from which the more negative feedback factor is, the deeper negative feedback will be, and the more frequency range will be in case of amplitude being flat, which is convenient for processing testing data. Magnetic feedback has the same effect on field signal and noise of amplifier input, not changing output SNR.

\section{Raising magnetic induction capacity}

\subsection{Raising the number of coil turns via section- wise winding}

Sensitivity can be enhanced via raising the number of coil turns, however it also results in the increase of coil inductance, resistance, distributive capacitance, reduction of resonance frequencies, and increase of resistance noise. Too much inductance may hardly match with amplifier, and amplifier noise current breeds more high frequency noise.

Section-wise winding without regulations can reduce inductance and distributive capacitance, as shown in Figure 4 the schematic of equivalence between inductance and distributive capacitance after sectioning. In case of total number of turns being certain, as the number of sections is increased, mutual induction and distributive capacitance will be reduced between sections, owing to which total induction and distributive capacitance can be reduced. In case of total number of turns being 11000 , simulation is carried out for inductance when $X=2-20$, the result is shown as Figure 5, from which the total induction can be decreased to $1 / 7$ of the original value in case of the number of sections from 2 to 20 [5].

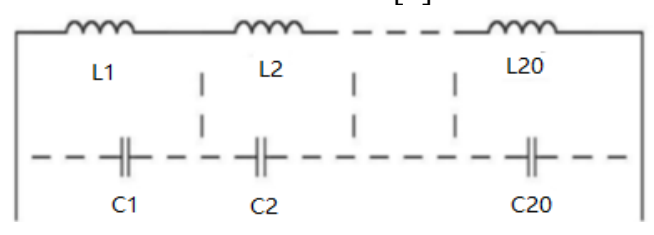

Fig. 4. Schematic of equivalence between inductance and distributive capacitance after sectioning.

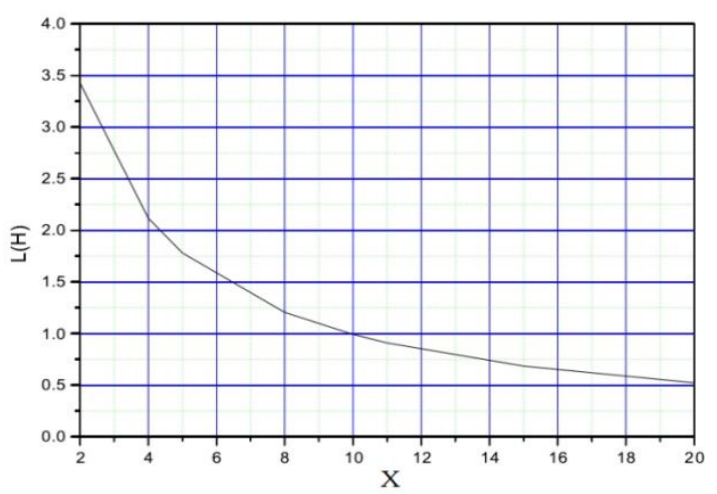

Fig. 5. The effect of sectioning on coil inductance.

\subsection{Raising induction capacity via core design}

Generally speaking, perm-alloy core has initial permeability $\mu_{c}$ up to 10000 plus, when the core is infinitely long or shaped round-loop, relative permeability $\mu_{r}$ is very close to $\mu_{c}$. But actually core with finite length is applied rather that infinite one, when it is magnetized by outside field, polarization charge, generated in the terminal, causes the decrease of inner-core magnetic field, reduction of relative permeability $\mu_{r}$, which depends on initial permeability of core and its geometric shape[6]: 


$$
\mu_{r}=\frac{\mu_{c}}{1+N_{d}\left(\mu_{c}-1\right)}
$$

In the formula $N_{d}$ is demagnetizing factor, related with the shape of core, $\mu_{c}$ being initial permeability.

$$
N_{d}=\left(\frac{d}{l}\right)^{2} \cdot\left[\ln \left(2 \frac{l}{d}\right)-1\right]
$$

In the formula $d$ is diameter of core, $l$ being its length. Generally, $\mu_{c}$ of core with high permeability is far more than 1 , and formula (3) can be simplified as:

$$
\mu_{r}=\frac{1}{N_{d}}
$$

Through the simulation via formula (5), Figure 6 shows the relation between draw ratio and effective permeability under different initial permeability. When $\mu_{c}$ is up to 100000 from 1000 , there is a rapid rise of $\mu_{r}$, whereas $\mu_{r}$ is no longer raised when $\mu_{c}$ is up to 50000, only raised rapidly as enhanced by draw ratio [6]. Therefore initial permeability of selected core shall be larger than 10000 , better up to 50000 , and perm-alloy initial permeability is $30000-50000$, which can better fulfil the requirements.

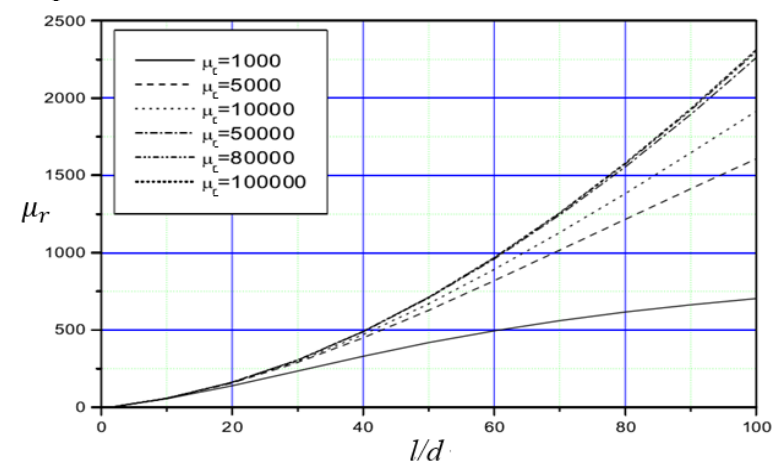

Fig. 6. The relation between draw ratio and effective permeability under different initial permeability.

Since coil length is not considered in formula (5), the calculated permeability is larger than actual value. During the design empirical formula is used to calculate effective permeability of iron core with finite length $\mu_{e}$ [5-6].

$$
\mu_{e}=\frac{\mu_{c} \cdot\left[1-0.255 \frac{l_{c}}{l}\right]}{1+0.975\left(\frac{S}{l^{2}}\right) \cdot\left[\ln \left(\sqrt{\frac{\pi l^{2}}{S}}\right)-1\right] \cdot\left(\mu_{c}-1\right)}
$$

In the formula $l_{c}$ refers to coil length, $S=d^{2}$. Effective permeability via empirical formula is $30 \%$ smaller than via that formula (5), which is closer to the actual value of core material.

Table 1. $\mu_{r}$ and $\mu_{e}$ under Different $l / d$

\begin{tabular}{|c|c|c|c|c|}
\hline $\boldsymbol{l}$ & $\mathbf{0 . 2 5} \mathbf{m}$ & $\mathbf{0 . 5 m}$ & $\mathbf{1 m}$ & $\mathbf{2 m}$ \\
\hline$l / d$ & 12.5 & 25 & 50 & 100 \\
\hline$\mu_{r}$ & 70.4 & 214.6 & 693 & 2326 \\
\hline$\mu_{e}$ & 50 & 167 & 561 & 1859 \\
\hline
\end{tabular}

$\mu_{r}$ and $\mu_{e}$ are calculated in case of $l / d$ being $12.5-100$ and $d=20 \mathrm{~mm}$, which shows as table 1 , effective permeability is risen to 3.25 times when core length is doubled.

In case of length of core being constant, relative permeability can be enhanced via reducing $d$ as per formula (3), however what matters to the performance is the product between relative permeability and sectional area via formula (1), which needs to be raised. Figure 7 shows curve between diameter of core and $\mu_{e} S$ in case of length of core being $0.75 \mathrm{~m}$, from which parameters of magnetic sensor are raised via increasing core sectional area, with performance being decreased rapidly under $\mathrm{d}$ less than $2.5 \mathrm{~mm}$, the effect of sectional area on $\mu_{e} S$ being little under $d$ more than $2.5 \mathrm{~mm}$. When $d$ is started to duplicate from $2.5 \mathrm{~mm}$, there is only $20 \%$ rise of electrical performance. If compactness of the sensor is especially considered, core diameter can be more than $2.5 \mathrm{~mm}$, however when structure strength is taken into account, generally $10 \mathrm{~mm}-20 \mathrm{~mm}$ will be better. If $d$ is to further rise, it will pose negative effect on the noise of sensor and its application since coil winding and core weight are too much [6]. Magnetic flow collector with large-area, added in both ends of core, will converge magnetic induction lines, which can easily improve effective permeability.

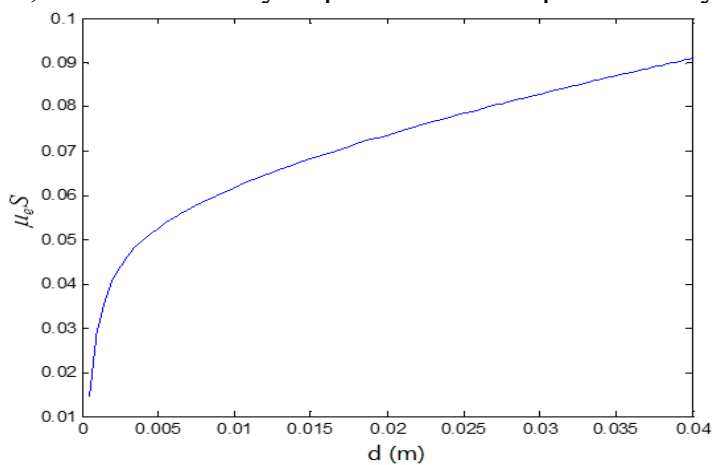

Fig. 7. Curve between diameter of core and $\mu_{e} S$ in case of length of core being $0.75 \mathrm{~m}$.

Based on the analysis above and project application, parameters are specified like core length being $1200 \mathrm{~mm}$, diameter $20 \mathrm{~mm}$, the total number of coil winding 40000, DC resistance being about $500 \Omega$, and coil inductance $300 \mathrm{H}$ around. Concerned with core material, aside from initial permeability, diameter and length, there is another index being core loss, which can be reduced via wafer piling up. However, core loss resistance at $10 \mathrm{kHz}-100 \mathrm{kHz}$ is generally 10 folds more than that at low frequency [5], nanocrystalline with far lower loss is adopted for highfrequency application.

\subsection{Low-noise pre-amplifier design}

Pre-amplifier design is crucial, since it boasts the function of amplifying output voltage signal, and adjusting feedback signal strength. In light of low noise and wide band features at low frequency, it is composed of lowfrequency chopping amplification channel and highfrequency amplification channel, which is shown as Figure 8 . The latter consists of amplifier A1 and high-pass filter F1, directly amplifying inducted voltage via operational amplifier, whereas the former consists of modulator M1, demodulator M2, amplifier A2, low-pass 
filter F2, and oscillation signal source G1, amplifying the signal before modulation at fixed frequency, followed by synchronized demodulation and output. 1/f noise effect can be avoided during weak signal amplification with chopping amplification being adopted at low frequency channel, and negative feedback chopper amplifier with low-offset is applied to reduce DC offset [3]. Figure 9 shows noise curve schematic during startup and shutoff of chopping amplifier, which finds that LF noise, is apparently reduced during startup of chopping amplifier [7].

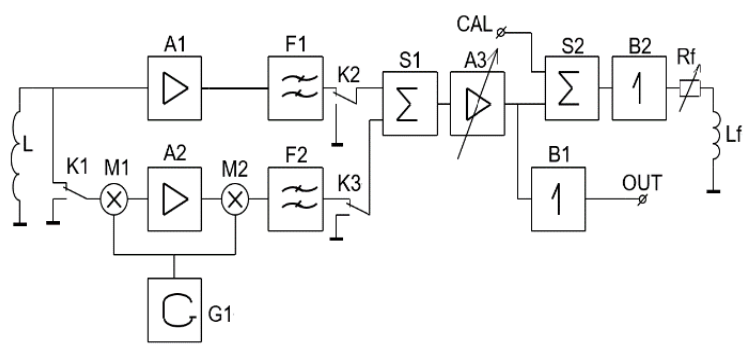

Fig. 8. Block diagram of preamplifier.

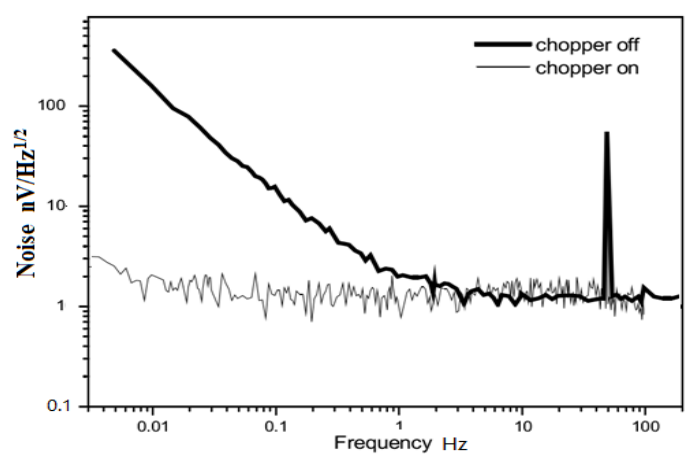

Fig. 9. Noise curve schematic during startup and shutoff of chopping amplifier.

Signals from coil $L$ are paralleled into input ends of both amplifiers with same amplifying coefficient, with filter F1 and F2 boasting same cut-off frequency and series, which makes the matching and addition between S1 signal and amplifier. Total gain of amplifier is 30000 times in an effort to realize switching factor of $1.6 \mathrm{f}(\mathrm{V} / \mathrm{nT})$. Chopping frequency is better to be $2 \mathrm{kHz}$, since lower frequency breeds overall high noise of chopping amplification, and higher one results in high upwarp below $0.001 \mathrm{~Hz}$, formulizing new $1 / \mathrm{f}$ noise at super-lowfrequency [7][8].

Figure 10 shows schematic block diagram of A1 and A2[2][9], in which differential amplifier with IFN860 JFET is adopted to realize low-voltage noise, low-current noise, and high-input impedance of pre-amplifier. Its input voltage is $1.8 \mathrm{nV} / \mathrm{Hz}^{1 / 2}$, and current being $10 \mathrm{fA} / \mathrm{Hz}^{1 / 2}$. Boasting the features of high-impedance and low distributive capacitance, the amplifier is suitable for wideband magnetic sensor. Noise voltage can be lower to $1 \mathrm{nV} / \mathrm{Hz}^{1 / 2}$ for amplifier with IF3602 [10], but its input distributive capacitance is high up to $300 \mathrm{pF}$, which is 20 times of IFN860 fit for low frequency application, whereas not suitable for high frequency application. If power supply of $\pm 12 \mathrm{~V}$ is adopted for the amplifier, power loss is less than $800 \mathrm{~mW}$; if power supply of $\pm 6 \mathrm{~V}$ is adopted for the amplifier, power loss is less than $400 \mathrm{~mW}$.

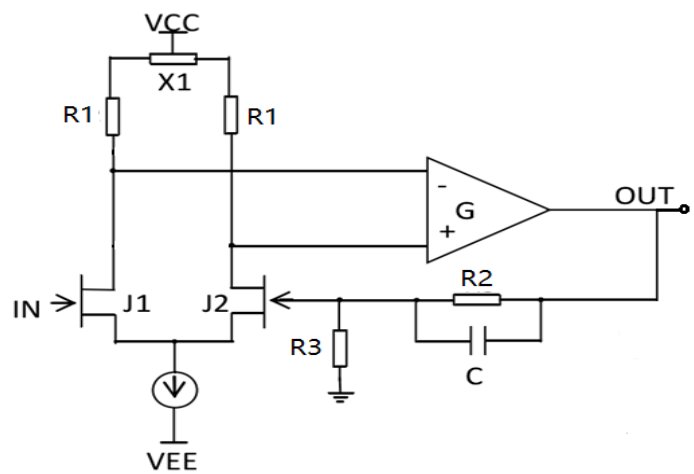

Fig. 10. Schematic block of JFET pair differential amplifier

\section{Noise analysis and testing}

Total noise voltage of magnetic sensor is mainly composed of heat noise of $R$, amplifier voltage noise $E_{n}$, noise of reaction between $I_{n}$ and $2 \pi f L$, and core loss noise $R_{s}$, of which the model is shown as Figure 11 [4][11][12].

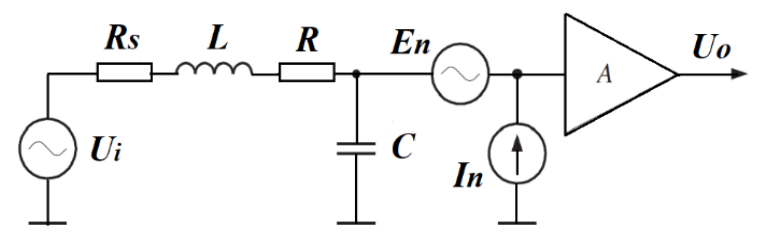

Fig.11. Noise model of magnetic sensor.

Total noise voltage $U_{n}$ is:

$$
U_{n}=\left(E_{n}{ }^{2}+4 k T\left(R+R_{s}\right)+\left(I_{n}{ }^{*} Z\right)^{2}\right)^{1 / 2}
$$

In the formula $k=1.38 \times 10^{-23}$ is Boltzman constant, $T=300$ being absolute temperature, $Z=2 \pi f L$ being coil impedance, and $R_{S}$ can be neglected at low frequency.

Table 2.Calculated value of voltage and magnetic field noise

\begin{tabular}{|c|c|c|c|c|}
\hline Frequency & $\mathbf{0 . 0 1 H z}$ & $\mathbf{1 H z}$ & $\mathbf{1} \mathbf{k H z}$ & $\mathbf{1 0 k H z}$ \\
\hline$I_{n} Z\left(\mathrm{nV} / \mathrm{Hz}^{1 / 2}\right)$ & 0 & 0.019 & 18.8 & 188 \\
\hline$U_{n}\left(\mathrm{nV} / \mathrm{Hz}^{1 / 2}\right)$ & 3.4 & 3.4 & 19.1 & 188 \\
\hline$B_{n}\left(\mathrm{nT} / \mathrm{Hz}^{1 / 2}\right)$ & $6.4^{*} 10^{-3}$ & $6.4 * 10^{-5}$ & $3.6^{*} 10^{-7}$ & $3.5^{*} 10^{-7}$ \\
\hline
\end{tabular}

Put the values into formula (7) such as heat noise of $R=500 \Omega$ being $2.9 \mathrm{nV} / \mathrm{Hz}^{1 / 2}, E_{n}$ being $1.8 \mathrm{nV} / \mathrm{Hz}^{1 / 2}, I_{n}$ being $10 \mathrm{fA} / \mathrm{Hz}^{1 / 2}$, and $L$ being $300 \mathrm{H}$, and noise voltage at different frequencies are shown as Table 2, which shows that noise current is mainly effective above $1 \mathrm{kHz}$, and noise field $B_{n}$ is calculated as per general formula[11]:

$$
B_{n}=U_{n} /\left(2 \pi f N S \mu_{e}\right)
$$

When amplifier gain $A=30000$ times and switching factor $K=1.6 \mathrm{fV} / \mathrm{nT}$ are certain, $B_{n}$ can be easier calculated according to $K=U_{n} * A / B_{n}$ :

$$
B_{n}=U_{n} * A / K
$$


Following sensor design, frequency response characteristic is shown as Figure 12 through actual test, with corner frequency being $0.06 \mathrm{~Hz}$, low-frequency switching factor being $K=1.6 \mathrm{fV} / \mathrm{nT}$, and high frequency being $0.12 \mathrm{~V} / \mathrm{nT}$. The comparison between noise test typical value and natural noise is shown as Figure 13, with sensor noise being $10^{-4} \mathrm{nT} / \mathrm{Hz}^{1 / 2}$ at $1 \mathrm{~Hz}, 10^{-6} \mathrm{nT} / \mathrm{Hz}^{1 / 2}$ at $100 \mathrm{~Hz}-1 \mathrm{kHz}$, which is $20 \mathrm{~dB}-40 \mathrm{~dB}$ lower than natural noise, and is increased a little at $10^{-4} \mathrm{~Hz}-10^{-3} \mathrm{~Hz}$ owing to undesirable noise of chopping amplifier. Flat response curve, wide frequency band, and low noise features are realized for the sensor at $0.0001 \mathrm{~Hz}-10 \mathrm{kHz}$.

Compared with calculated value, typical value of noise test is compatible under $2 \mathrm{kHz}$, whereas larger above $2 \mathrm{kHz}$ since lack of core high frequency loss parameter and undesirable testing environment.

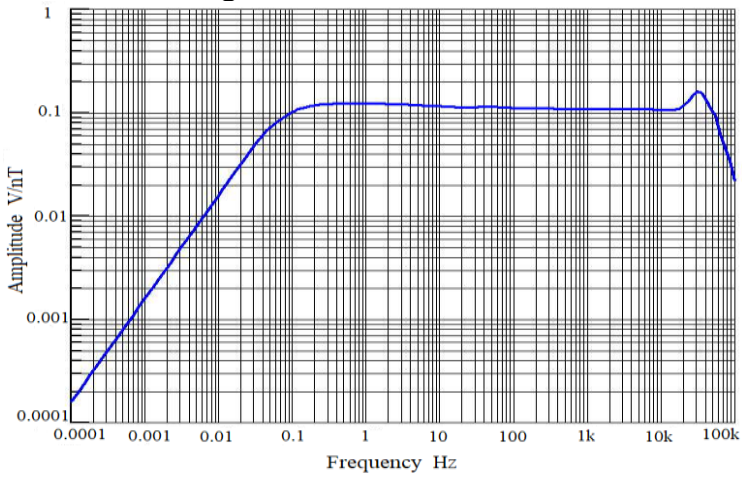

Fig.12. Frequency response feature of magnetic sensor.

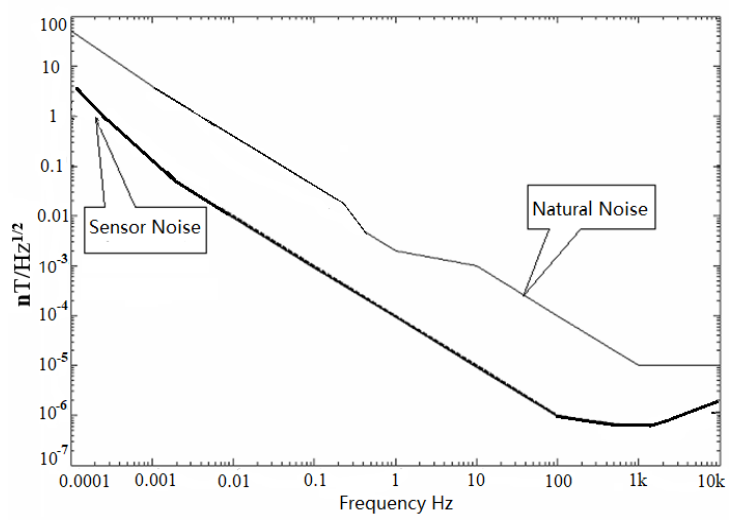

Fig.13. Comparison between typical sensor noise and natural background noise.

\section{Conclusion}

Coil induction capacity is enhanced through optimization of core high-permeability material selection and coil design. In order to simultaneously reach ultra-low noise and wide frequency band, this paper introduces low-noise amplifier in combination of JFET differential pair lowfrequency chopping amplification channel and highfrequency amplification channel, along with magnetic feedback technology. Test result shows noise at $100 \mathrm{~Hz}-$ $1 \mathrm{kHz}$ can as low as to $10^{-6} \mathrm{nT} / \mathrm{Hz}^{1 / 2}$, which reach SQUID (superconducting quantum interference device) level. Since wide frequency band $0.0001 \mathrm{~Hz}-10 \mathrm{kHz}$ and ultralow noise features are realized, single magnetic sensor covers operation frequencies of both MT and AMT, which can better meet the requirements of project site.

Future work will be aimed at optimizing less than $0.001 \mathrm{~Hz}$ and higher band noise. The follow-up plan is to implement the research on the mechanism and treatment method of the extremely low noise of the chopper amplifier, and to carry out the research on the highfrequency characteristics of the high-permeability magnetic core material and the high-frequency current noise of the converter, improve the sensor design, and improve the extreme sensitivity of the sensor at extremely low and high frequency.

\section{References}

1. Y. Zhao, X. Chen, G. Wang, etc. Signal Conditioning Circuit Study of Frequency Domain Inductive Magnetic Sensor, Geophysical and Chemical Computation Technology,3,199-205(2016)

2. W. Huang, The Research on Compact Winding Inductive Sensor with Hollow-core, Master Degree Paper of Jilin University (2018)

3. J. Li, The Research on DC Offset Suppression of Chopper Amplifying Circuit, Master Degree Paper of Jilin University (2019)

4. X. Liu, W. Zhu, B. Yan, etc., Inductive Sensor for Lightning field measurement, Apparatus Technologies and Sensor, 1, 5-8 (2020)

5. T. Qing,Transmission Characteristic Study of Inductive Magnetic Negative Feedback Sensor, Master Degree Paper of Jilin University (2012)

6. W. Li, Y. Liu, Y. Fan, Micro-sized Wide-frequency band Magnetic Sensor Design, Ship Electronic Engineering, 3,129-133(2012)

7. Q. Zhao, Design and Realization of Inductive Magnetic Sensor at $0.003-10 \mathrm{kHz}$, Master Degree Paper of Jilin University (2013)

8. H. Shi, Y. Wang, J. Lin, Optimal Design of Lownoise Induction Magnetometer in $1 \mathrm{mHz}-10 \mathrm{kHz}$ Utilizing Paralleled Dual-JFET Differential Preamplifier, IEEE Sensors Journal,2537928,1-7(2016)

9. Japheth E. Pakaree, Viranjay M. Srivastava, Realization with fabrication of double-gate MOSFET based, differential amplifier, Microelectronics Journal 91 (2019)

10. K. Liu , Ultralow Noise Preamplifier and Optimization Method for Induction Magnetometers, IEEE SENSORS JOURNAL, VOL.15,32933300(2015)

11. A. Grosz, E. Paperno,A. O. of L. S. C. M., IEEE SENSORS JOURNAL, VOL. 12,2719-2723(2012)

12. M. Abdul Mubin, A. Marzuki, A Low-Noise Amplifier Utilizing Current-Reuse Technique and Active Shunt Feedback for MedRadio Band Applications, International Journal of Electrical and Electronic Engineering \& Telecommunications (2020) 